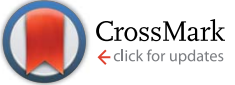

Cite this: RSC Adv., 2016, 6, 63058

\title{
Graphene material prepared by thermal reduction of the electrochemically synthesized graphite oxide
}

\begin{abstract}
B. Gurzęda, ${ }^{a}$ P. Florczak, ${ }^{\text {b } M . ~ W i e s n e r, ~}{ }^{\text {bc }}$ M. Kempiński, ${ }^{\text {bd }}$ S. Jurga ${ }^{\text {be }}$ and P. Krawczyk ${ }^{\star a}$
In the present work we demonstrate a simple and effective way to produce bulk quantities of graphene material. For the first time, graphite oxide (GO), synthesized by electrochemical treatment of natural graphite in $\mathrm{HClO}_{4}$ aqueous solution, was used to obtain thermally exfoliated-reduced graphite oxide (TRGO). Herein, GO was thermally exfoliated and reduced at $500{ }^{\circ} \mathrm{C}$ in air, giving the final product of TRGO. Due to shock treatment, the volume of the synthesized TRGO drastically increased compared to the starting GO. Furthermore, the exfoliation process resulted in a significant decrease in the concentration of oxygen functionalities. The choice of GO exfoliation temperature was preceded by thermogravimetric analysis (TGA). TRGO was characterized using X-ray diffraction (XRD), Raman spectroscopy, X-ray photoelectron spectroscopy (XPS), scanning electron microscopy (SEM) and atomic force microscopy (AFM) analysis.
\end{abstract}

Received 27th April 2016

Accepted 22nd June 2016

DOI: $10.1039 / c 6 r a 10903 a$

www.rsc.org/advances

The method applied in the production of graphene materials, as well as the specific conditions, have a strong effect on their characteristics, enabling their different applications in i.e. energy storage devices, electronics, catalyst supports, sensors, biomedicines and composite materials. ${ }^{12,17,18}$

One of the most promising methods for the preparation of bulk quantities of graphene material is the thermal exfoliation of GO accompanied by its reduction. ${ }^{19,20}$ During these processes, most of the oxygen functionalities are removed from the graphitic structure. Simultaneously, the volume of thermally exfoliated-reduced graphite oxide (TRGO), the product of these processes, significantly increases compared to that of the starting material. ${ }^{21} \mathrm{GO}$ can be exfoliated and reduced yielding TRGO by a rapid thermal treatment at $1050^{\circ} \mathrm{C}$ in air or an argon atmosphere. ${ }^{21-24}$ TRGO is also produced by the heating of GO at elevated temperatures up to $1000{ }^{\circ} \mathrm{C}$ under an inert gas flow (nitrogen or argon) ${ }^{13,14,25}$ or in the presence of hydrogen, used as a reducing agent. The use of a hydrogen atmosphere enables the entire removal of oxygen functional groups from $\mathrm{GO}^{20} \mathrm{On}$ a much smaller scale, the exfoliation-reduction process of GO can be carried out using microwave treatment. ${ }^{26,27}$ The properties of TRGO depend on many factors; among these, the method used, the properties of the applied precursor and conditions (atmosphere, treatment time, temperature) seem to be the most important. ${ }^{1,12,13,24,26}$ As can be seen from the literature data, most of the research works are concerned with the exfoliationreduction process of chemically prepared GO. ${ }^{14,19-26}$

The present work describes a modification of the method to produce bulk quantities of graphene material. Based on our current knowledge, this is the first time that GO, synthesized by the deep anodic oxidation of graphite in perchloric acid, was used as a precursor in order to obtain TRGO. The process of GO 
exfoliation was carried out at relatively low temperature under an air atmosphere. The synthesized graphene material was characterized by various techniques, such as X-ray diffraction (XRD), Raman spectroscopy, scanning electron microscopy (SEM) and X-ray photoelectron spectroscopy (XPS). Additionally, to prove the formation of multi-layer graphene, the characterization of TRGO by atomic force microscopy (AFM) was performed.

\section{Experimental}

\subsection{Preparation of GO}

In order to obtain graphite oxide, natural graphite flakes (purity 99.5\%, flake size 170-283 $\mu \mathrm{m}$, Graphit Kropfmühl, Germany), playing the role of the working electrode, was electrochemically oxidized in an aqueous solution of $8 \mathrm{M} \mathrm{HClO}_{4}$ (purity $71 \%$, Merck, Germany) using a linear sweep voltammetry method (LSV). Platinum wire (purity 99.9\%, $1 \mathrm{~mm}$ diameter, Goodfellow, United Kingdom) was used as a counter electrode, whereas $\mathrm{Hg} / \mathrm{Hg}_{2} \mathrm{SO}_{4} / 1 \mathrm{M} \mathrm{H}_{2} \mathrm{SO}_{4}$ was a reference electrode. LSV measurements were performed in the potential range from the rest potential of the electrode $\left(E_{\mathrm{R}}\right)$ to $1.4 \mathrm{~V}$ with the scan rate equal to $0.01 \mathrm{mV} \mathrm{s}^{-1}$. The details of GO formation can be found in our previous work. ${ }^{28}$

\subsection{Thermal reduction of GO}

TRGO was prepared through the thermal treatment of the previously synthesized GO. The target product is the result of the thermal reduction of GO accompanied by its exfoliation. A ceramic crucible containing GO was promptly inserted into the muffle furnace adjusted to $500{ }^{\circ} \mathrm{C}$. Heat treatment was carried out in an air atmosphere for $4 \mathrm{~min}$. After the process, the asprepared TRGO was rapidly removed from the furnace and allowed to cool in air to reach room temperature.

\subsection{Preparation of sample for AFM}

In order to perform AFM measurements, a colloidal suspension of the synthesized material was prepared. First, a mixture of TRGO (concentration $0.1 \mathrm{mg} \mathrm{ml} \mathrm{ml}^{-3}$ ) in dimethylformamide (DMF) (purity 99.8\%, POCH, Poland) was sonicated for 1 hour. Consecutively, the prepared suspension was centrifuged at 4000 rpm for $15 \mathrm{~min}$. The gathered supernatant was transferred into a silicon substrate and kept for solvent evaporation.

\subsection{Instrumentation}

The process of GO preparation was carried out using an Autolab PGSTAT 302N potentiostat/galvanostat (Metrohm, Netherlands) by the LSV technique. The exfoliation-reduction of GO was performed in a Nabertherm LE 14/11/P300 muffle furnace (Nabertherm, Germany). TRGO dispersion was reached by sonication using an ultrasonic bath SONOREX RK 52, HFFrequency $35 \mathrm{kHz}$ (Bandelin, Germany). During the thermogravimetric analysis (TGA) (Setaram France), the GO sample was heated up to $800{ }^{\circ} \mathrm{C}$ with a heating rate of $5{ }^{\circ} \mathrm{C} \mathrm{min}^{-1}$. The morphology of TRGO was characterized using a JSM-7001F scanning electron microscope (SEM) (Jeol, Japan), whereas structural characterization was carried out by X-ray diffraction (XRD) and Raman spectroscopy. XRD analysis was carried out on a Empyrean diffractometer (PANalytical, Netherlands) using $\mathrm{Cu} \mathrm{K} \alpha$ radiation $(1.54 \AA)$, a reflection-transmission spinner (sample stage) and a PIXcel 3D detector, operating in the BraggBrentano geometry. 2 Theta scans were recorded at room temperature with angles of 2 Theta ranging from $4^{\circ}$ to $60^{\circ}$, with a step size of $0.006^{\circ}$ in a continuous scan mode. Raman spectra were recorded using an inVia Raman Microscope (Renishaw, United Kingdom) with a $514 \mathrm{~nm}$ laser excitation source. In order to examine the concentration of surface oxygen functionalities, X-ray photoelectron spectroscopy (XPS) was performed. XPS spectra were obtained with a Sphera II photoelectron energy analyzer (Scienta Omicron, Germany) with the monochromatized $\mathrm{Al} \mathrm{K} \alpha$ X-ray source mounted inside the UHV system. The surface topography of TRGO was investigated by an atomic force microscope Bruker Dimension Icon (Bruker, Germany) using a standard tapping mode with a scan rate of $0.1 \mathrm{~Hz}$. The thickness and size distributions of TRGO were estimated using Gwyddion software. ${ }^{29}$

\section{Results and discussion}

Thermal stability of the electrochemically synthesized GO was investigated by thermogravimetric analysis (TGA). The results of this measurement allow the determination of the temperature of GO exfoliation. The thermogravimetric curve shows two major effects associated with the mass loss (see Fig. 1). The first one at $230{ }^{\circ} \mathrm{C}$ corresponds to the removal of most of oxygencontaining functional groups from the GO structure (almost $32 \%$ loss of starting weight). The latter decrease in sample weight (above the temperature of $550{ }^{\circ} \mathrm{C}$ ) is associated with the thermal decomposition of GO. When the temperature of $800{ }^{\circ} \mathrm{C}$ is reached, the entire sample is burned out. In order to provide appropriate conditions for the exfoliation-reduction of GO, thereby avoiding its thermal decomposition, the temperature

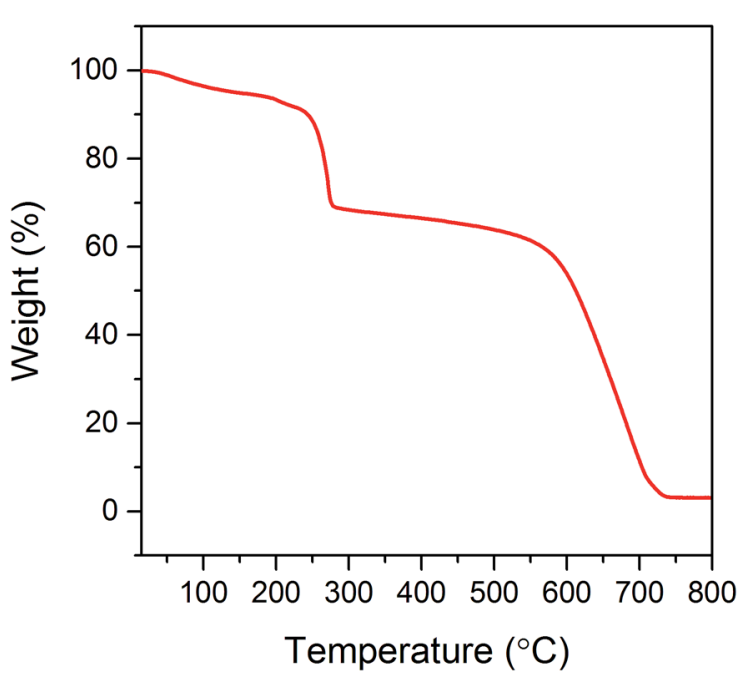

Fig. 1 Thermogravimetric analysis of GO under an air atmosphere with a heating rate of $5^{\circ} \mathrm{C} \mathrm{min}^{-1}$. 
was adjusted to $500{ }^{\circ} \mathrm{C}$. From the literature data, it is known that most of the oxygen functionalities within the GO start to decompose at a temperature of around $250{ }^{\circ} \mathrm{C} . .^{\mathbf{1 1 3}, 24}$ Therefore, due to the thermal shock treatment, at the selected temperature $\left(500{ }^{\circ} \mathrm{C}\right)$, an abrupt decomposition of oxide functionalities chemically bonded to the graphene layers occurs. The gaseous products of the above-mentioned decomposition $\left(\mathrm{CO}_{2}, \mathrm{CO}\right.$ and water) are accumulated within the interlayer spaces of the graphitic structure. The increasing amount of produced gases leads to the increase in pressure. When the mechanical strength of the graphene layers is exceeded, the gaseous products of GO decomposition are abruptly thrown out from the structure. The violent character of this process results in some deformation of the graphene layers, which is accompanied by a tremendous increase in the sample volume.

Fig. 2a confirms that the thermal shock treatment of GO performed at $500{ }^{\circ} \mathrm{C}$ in air atmosphere yields TRGO of a significantly increased volume. Owing to the employed procedure, 90 $\mathrm{mg}$ of TRGO were synthesized from about $150 \mathrm{mg}$ of GO. The loss of material due to thermal treatment is likely associated with the decomposition of oxygen groups followed by their removal from the graphene structures. The violent character of this process causes a significant disordering in the TRGO structure. ${ }^{30}$ The SEM images shown in Fig. $2 b-d$ display that the most of the graphene layers pertaining to the synthesized material are crumpled and creased. However, some graphene sheets are also observed.

The XRD patterns of graphite, GO and TRGO are shown in Fig. 3. The pattern of graphite (Fig. 3a) exhibits characteristic sharp diffraction peaks at a 2 Theta value of $26.6^{\circ}$ with a $d$ spacing of $3.35 \AA$ and a 2 Theta value of $54.8^{\circ}$ with a $d$ spacing of $1.68 \AA$, respectively. Curve b, recorded for GO, displays changes in the graphite structures caused by its electrochemical oxidation in $8 \mathrm{M} \mathrm{HClO}_{4}$. Due to the formation of oxygen groups on the graphite surface, the $d$ spacing increased from 3.35 to

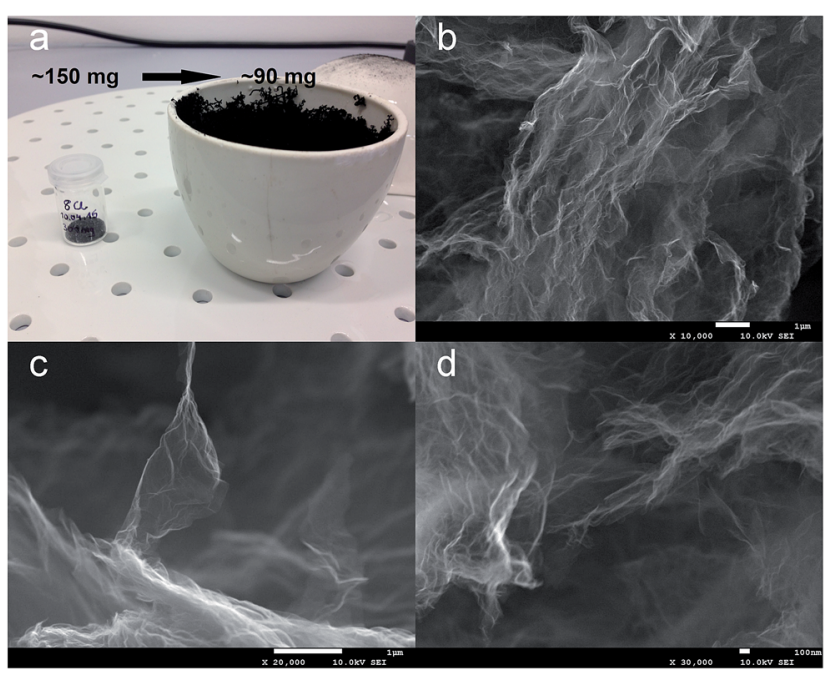

Fig. 2 Digital photo of GO before thermal treatment and resulting TRGO (a), SEM images of synthesized TRGO recorded under different magnifications $(b-d)$.

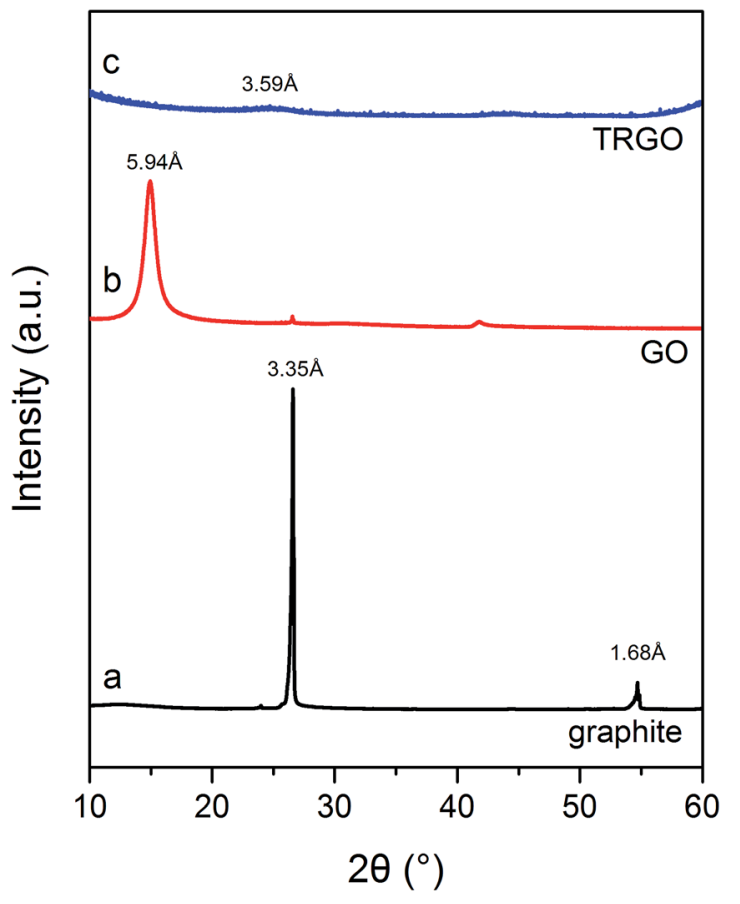

Fig. 3 XRD patterns of graphite (a), GO (b) and TRGO (c).

$5.94 \AA$ A thus revealing the GO formation. The appearance of the GO structure is also proved by the diffraction peak at $14.9^{\circ}$ seen on curve b. The XRD pattern of TRGO (curve c) exhibits a small diffraction peak corresponding to an average $d$ spacing of 3.59 A. This indicates that during the exfoliation-reduction process, graphene layers of GO are transformed into non-crystalline graphene stacks with random interlayer spaces. ${ }^{13,24,31}$ The changes in the GO structure due to the thermal treatment yielding TRGO are also provided from Raman spectroscopy analysis.

Raman spectroscopy is a technique commonly used to examine the degree of disorder of carbon materials. Fig. 4a shows two peaks, positioned at 1352 and $1580 \mathrm{~cm}^{-1}$, which are typical for a graphite structure. The first one, denoted as a D band peak, is attributed to structural defects, edges, as well as oxidation of the graphite surface. ${ }^{3,34}$ The latter peak (G band peak), is sharp and intense. This signal is associated with the radial $\mathrm{C}-\mathrm{C}$ stretching mode of $\mathrm{sp}^{2}$ bonded carbon. ${ }^{34,35}$ The Raman spectrum of GO (Fig. 4b) shows a significant increase in intensity of the $\mathrm{D}$ band peak, with a maximum around 1344 $\mathrm{cm}^{-1}$, whereas the $\mathrm{G}$ band peak becomes flattened. The formation of GO also results in a peak shift. For GO, the D band peak is positioned at $1344 \mathrm{~cm}^{-1}$, whereas the $\mathrm{G}$ band peak is at $1589 \mathrm{~cm}^{-1}$. The $I_{\mathrm{D}} / I_{\mathrm{G}}$ ratio $\left(I_{\mathrm{D}}-\right.$ intensity of $\mathrm{D}$ band peak, $I_{\mathrm{G}}-$ intensity of $\mathrm{G}$ band peak) provides information on the defect density of the graphitic structure. ${ }^{32}$ It can observed that as a consequence of the transformation of graphite into GO, the $I_{\mathrm{D}} /$ $I_{\mathrm{G}}$ ratio significantly increases from 0.07 to 0.75 , indicating a significant worsening of the structural order. Simultaneously, due to the oxidation of graphite in $\mathrm{HClO}_{4}$, oxygen functionalities are intensively formed, causing a decrease in the 


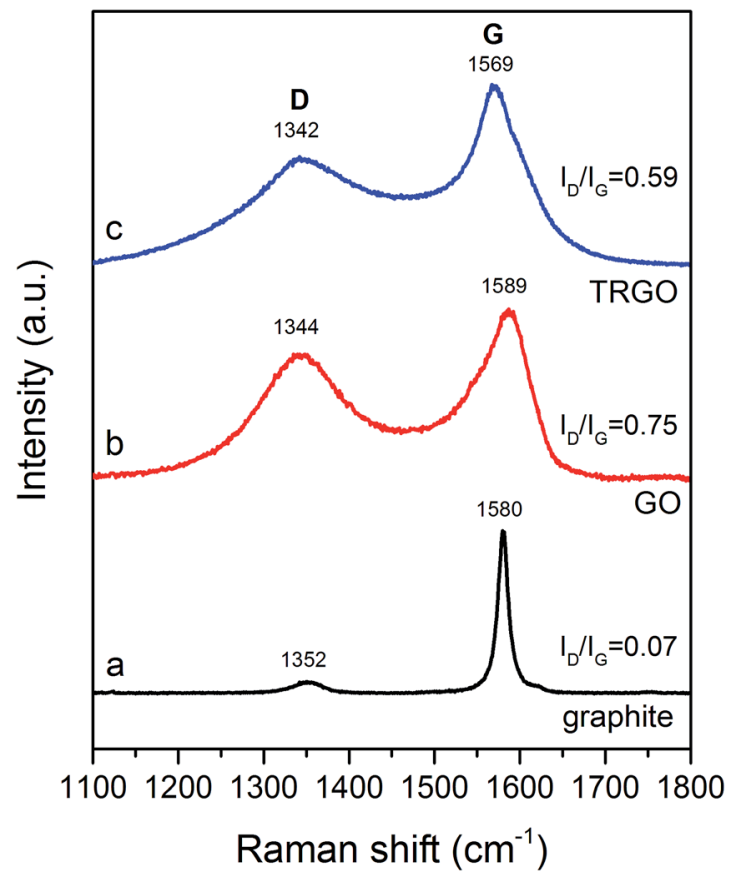

Fig. 4 Raman spectra of graphite (a), GO (b) and TRGO (c).

concentration of $\mathrm{sp}^{2}$-hybridized $\mathrm{C}-\mathrm{C}$ bonds. ${ }^{20,34,36,37}$ After the thermal treatment of GO, yielding TRGO, the $I_{\mathrm{D}} / I_{\mathrm{G}}$ ratio decreases to 0.59 (Fig. 4c). The intensity of the $\mathrm{D}$ band peak for the TRGO sample is lower in comparison with GO (Fig. 4b). On the other hand, the $\mathrm{G}$ band peak is slightly sharper than that for GO and is shifted to $1569 \mathrm{~cm}^{-1}$. Such behaviour can be likely elucidated in terms of the decomposition of the oxygen groups chemically bounded to graphene layers, as well as the recovery of the $\mathrm{sp}^{2}$-hybridized $\mathrm{C}-\mathrm{C}$ bonds in the synthesized TRGO. ${ }^{38,39}$

The changes in chemical composition of the GO surface resulting from the thermal exfoliation-reduction process were examined by XPS analysis. Fig. 5 shows the deconvoluted XPS spectra of the C1s region recorded for GO and TRGO, respectively. Information on the total content of oxygen and carbon, the positions of the deconvoluted C1s peak, as well as the concentration of oxygen containing functional groups, is presented in Table 1. As expected, the thermal treatment of GO significantly decreases the total oxygen content. According to the literature data, ${ }^{\mathbf{4 0 , 4 1}}$ the
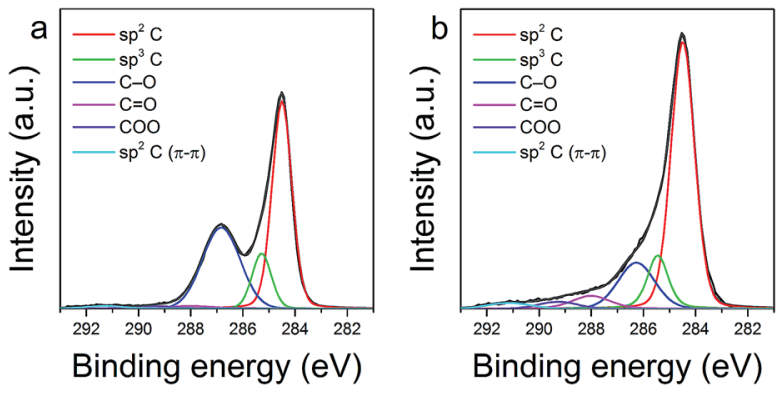

Fig. 5 C1s XPS spectra of GO (a), and TRGO (b).
Table 1 The XPS results for GO and TRGO

\begin{tabular}{|c|c|c|c|c|}
\hline \multicolumn{4}{|c|}{ GO } & \multirow{2}{*}{ TRGO } \\
\hline At $\%$ & \multirow{2}{*}{\multicolumn{2}{|c|}{$90.75 \%$}} & & \\
\hline $\mathrm{C}$ & & & & $95.14 \%$ \\
\hline $\mathrm{O}$ & \multicolumn{2}{|c|}{$9.25 \%$} & & $4.86 \%$ \\
\hline Surface groups & Peak (eV) & $\begin{array}{l}\text { Content } \\
(\%)\end{array}$ & Peak $(\mathrm{eV})$ & $\begin{array}{l}\text { Content } \\
(\%)\end{array}$ \\
\hline $\mathrm{sp}^{2} \mathrm{C}(\pi-\pi)$ & 291.2 & 1.50 & 291.1 & 2.27 \\
\hline $\mathrm{COO}$ & 289.3 & 1.33 & 289.3 & 2.50 \\
\hline $\mathrm{C}=\mathrm{O}$ & 288.0 & 1.85 & 288.0 & 5.16 \\
\hline $\mathrm{C}-\mathrm{O}$ & 286.8 & 34.20 & 286.4 & 16.20 \\
\hline $\mathrm{sp}^{3} \mathrm{C}$ & 285.3 & 12.38 & 285.4 & 12.05 \\
\hline $\mathrm{sp}^{2} \mathrm{C}$ & 284.5 & 48.74 & 284.5 & 61.82 \\
\hline
\end{tabular}

decomposition of epoxy and alkoxy groups, illustrated by the decrease in intensity of the peak at $286.5 \mathrm{eV}$, is responsible for the aforementioned decrement. On the other hand, it should be emphasized, that the thermal treatment of GO performed at $500{ }^{\circ} \mathrm{C}$ in an air atmosphere increased the concentration of some oxygen groups (carbonyl at $288.0 \mathrm{eV}$ and carboxyl at $289.3 \mathrm{eV}){ }^{40-43}$ Another result of the thermal exfoliation-reduction is associated with the significant increase in the contribution of $\mathrm{sp}^{2} \mathrm{C}$ (from 48.74 to $61.83 \%$ ). This effect is likely related with the decomposition of oxygen functionalities (alkoxy and epoxy groups), with the simultaneous recovery of $\mathrm{sp}^{2}$ hybridized carbon species. The XPS results are in agreement with the data presented in the Raman analysis.

Additional information on the structural properties of the synthesized TRGO, especially its topography, is provided by atomic force microscopy (AFM) analysis. The preparation of the TRGO sample for AFM investigations included the formation of a TRGO/dimethylformamide (DMF) mixture which underwent sonication followed by centrifugation. AFM observations of TRGO were performed with the use of a silicon substrate. The AFM image of a single graphene sheet with a lateral size of a few $\mu \mathrm{m}$ and its height profile are presented in Fig. 6. As can be seen from Fig. 6a, due to the thermal shock treatment of the GO, the obtained TRGO material has a wrinkled and creased structure. The height profile (Fig. 6b) shows that the graphene sheet is unevenly distributed on the silicon substrate. The height of the TRGO sheet, measured at the lowest point (see Fig. 6b), is equal to $2.2 \mathrm{~nm}$, which corresponds to multi-layer graphene (6-7 layers). ${ }^{44}$ To acquire additional information on the quality of the TRGO sample, as well as on its size distribution, a statistical analysis of almost 900 graphene sheets was performed (see Fig. 6c-e). As can be seen in Fig. 6d, most of the prepared graphene flakes have an equivalent radius of around $150 \mathrm{~nm}$. On the other hand, flakes of a higher radius are also observed. The thickness of graphene sheets ranged from 1 to $5 \mathrm{~nm}$. Only a small amount of graphene layers are thicker than $5 \mathrm{~nm}$ (Fig. 6e). 
a

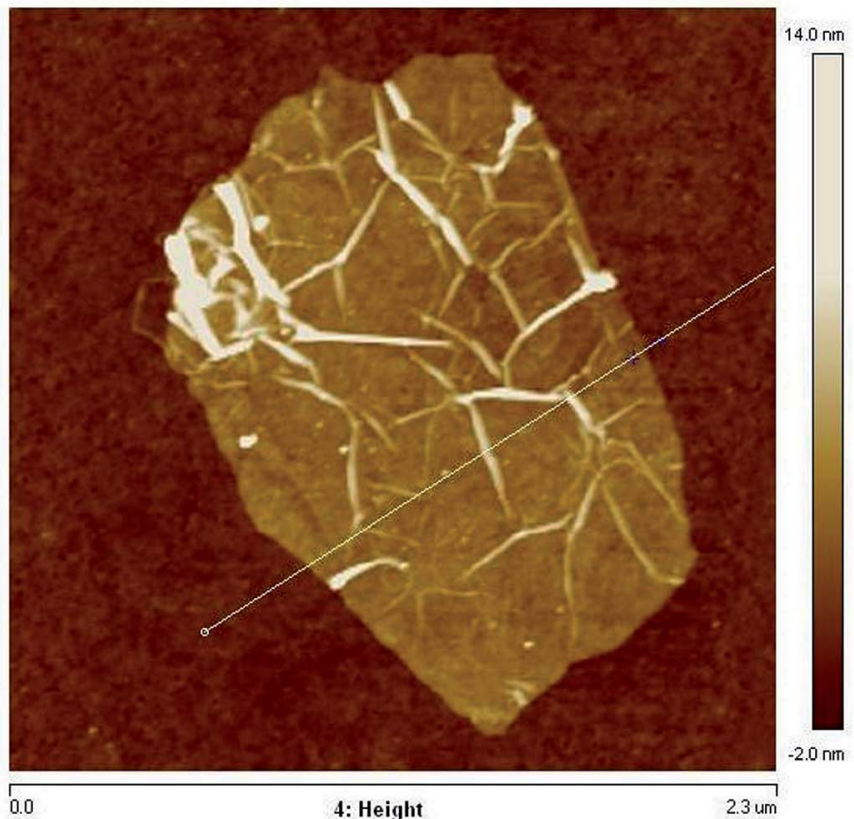

b

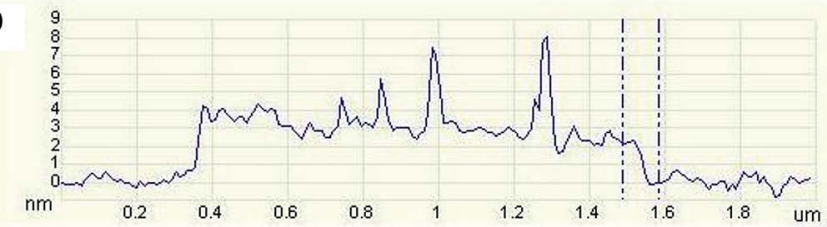

C

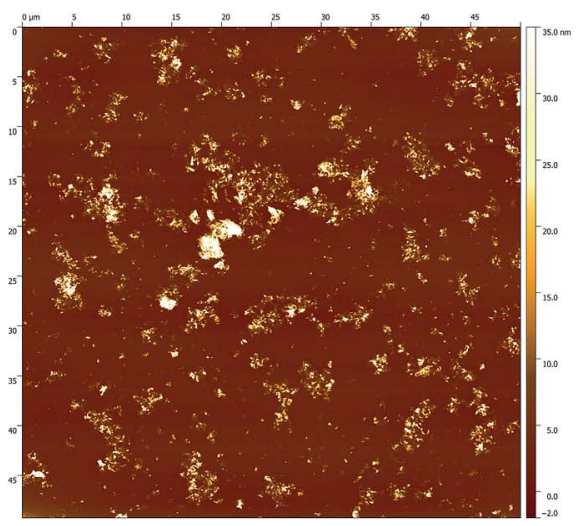

d

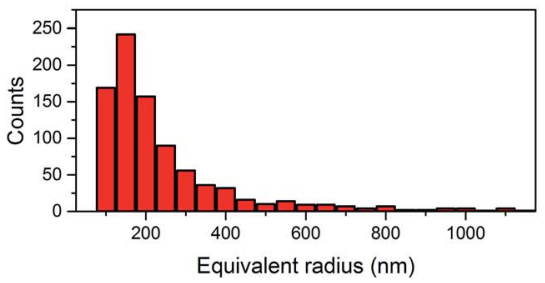

e

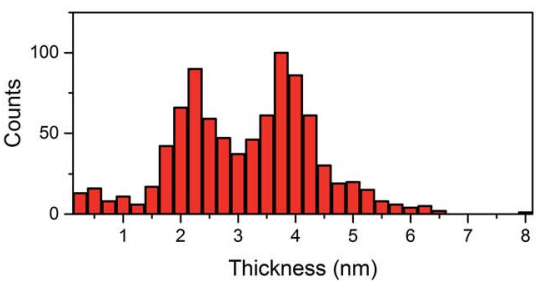

Fig. 6 Topography of a graphene-like sheet (a) and its height profile (b), micrograph of a $50 \times 50 \mu$ m area of a silicon substrate supporting graphene material flakes (c), flake equivalent radius distribution of 876 graphene material flakes (d) and its thickness distribution (e).

\section{Conclusions}

Our investigations undoubtedly reveal that bulk quantities of graphene material can be synthesized by the thermal exfoliation-reduction of GO prepared by the electrochemical oxidation of graphite in $8 \mathrm{M} \mathrm{HClO}_{4}$. The disappearance of the crystalline structure due to the thermal treatment of GO was confirmed by the XRD and Raman spectroscopy measurements. The results of the XPS analysis indicate the partial reduction of GO, caused by the decomposition of epoxy and alkoxy groups. On the other hand, the air atmosphere used during the thermal treatment of GO impacts on its partial oxidation resulting in a slight increment in the concentration of carbonyl and carboxyl groups. The thermal decomposition of oxygen functionalities followed by abrupt release of gaseous products from the GO structure resulted in an enormous increase in the volume of TRGO formed, yielding the exfoliation. The graphene layers within the obtained graphene material are wrinkled and creased with numbers of structural defects. The results of AFM analysis confirmed that to obtain multi-layer graphene, the additional treatment of TRGO with ultrasonication in DMF is necessary.

\section{Acknowledgements}

The authors thank Dr Barbara Peplińska of NanoBioMedical Centre, Adam Mickiewicz University for measurements and helpful discussion regarding SEM. The research was supported by National Centre for Research and Development under the research grant "Nanomaterials and their application to biomedicine", contract number PBS1/A9/13/2012. Mateusz Kempiński and Maciej Wiesner thank the National Center of Science for financial support, Grants No. DEC-2013/09/B/ST4/ 03711 and DEC-2014/15/B/ST3/02927.

\section{Notes and references}

1 S. Stankovich, D. A. Dikin, G. H. B. Dommett, K. M. Kohlhaas, E. J. Zimney, E. A. Stach, R. D. Piner, S. T. Nguyen and R. S. Ruoff, Nature, 2006, 442, 282.

2 M. Yi and Z. Shen, J. Mater. Chem. A, 2015, 3, 11700.

3 Y. Zhu, S. Murali, W. Cai, X. Li, J. W. Suk, J. R. Potts and R. S. Ruoff, Adv. Mater., 2010, $22,1$.

4 Z. Li, M. He, D. Xu and Z. Liu, J. Photochem. Photobiol., C, 2014, $18,1$.

5 C. K. Chua and M. Pumera, Chem. Soc. Rev., 2014, 43, 291. 
6 S. Gadipelli and Z. X. Guo, Prog. Mater. Sci., 2015, 69, 1.

7 A. Bianco, H. M. Chen, T. Enoki, Y. Gogotsi and R. H. Hurt, Carbon, 2013, 65, 1.

8 W. Fu, J. Kiggans, S. H. Overbury, V. Schwartz and C. Liang, Chem. Commun., 2011, 74, 5265.

9 S. R. Dhakate, N. Chauhan, S. Sharma, J. Tawale, S. Singh, P. D. Sahare and R. B. Mathur, Carbon, 2011, 49, 1946.

10 P. Krawczyk and B. Gurzęda, J. Solid State Electrochem., 2016, 20, 361 .

11 C. T. J. Low, F. C. Walsh, M. H. Chakrabarti, M. A. Hashim and M. A. Hussain, Carbon, 2013, 54, 1.

12 T. D. Dao and H. M. Jeong, Mater. Res. Bull., 2015, 70, 651.

13 C. Botas, P. Álvarez, C. Blanco, R. Santamaría, M. Granda, M. D. Gutiérrez, F. Rodríguez-Reinoso and R. Menéndez, Carbon, 2013, 52, 476.

14 H. Xian, T. Peng, H. Sun and J. Wang, Nano-Micro Lett., 2015, $7,17$.

15 A. Reina, X. Jia, J. Ho, D. Nezich, H. Son, V. Bulovic, M. S. Dresselhaus and J. Kong, Nano Lett., 2008, 9, 30.

16 D. V. Kosynkin, A. L. Higginbotham, A. Sinitskii, J. R. Lomeda, A. Dimiev, B. K. Price and J. M. Tour, Nature, 2009, 458, 872.

17 P. Avouris and C. Dimitrakopoulos, Mater. Today, 2012, 15, 86.

18 V. Georgakilas, M. Otyepka, A. B. Bourlinos, V. Chandra, N. Kim, K. C. Kemp, P. Hobza, R. Zboril and K. S. Kim, Chem. Rev., 2012, 112, 6156.

19 X. Gao, J. Jang and S. Nagase, J. Phys. Chem. C, 2010, 114, 832. 20 D. Zhan, Z. Ni, W. Chen, L. Sun, Z. Luo, L. Lai, T. Yu, A. T. S. Wee and Z. Shen, Carbon, 2011, 49, 1362.

21 H. C. Schniepp, J. L. Li, M. J. McAllister, H. Sai, M. HerreraAlonso, D. H. Adamson, R. K. Prud'homme, R. Car, D. A. Saville and I. A. Aksay, J. Phys. Chem. B, 2006, 110, 8535. 22 S. R. C. Vivekchand, C. S. Rout, K. S. Subrahmanyam, A. Govindaraj and C. N. R. Rao, J. Chem. Sci., 2008, 120, 9. 23 O. Akhavan, Carbon, 2010, 48, 509.

24 M. J. McAllister, J. L. Li, D. H. Adamson, H. C. Schniepp, A. A. Abdala, J. Liu, M. Herrera-Alonso, D. L. Milius, R. Car, R. K. Prud'homme and I. A. Aksay, Chem. Mater., 2007, 19, 4396.
25 B. Zhao, P. Liu, Y. Jiang, D. Pan, H. Tao, H. Song, T. Fang and W. Xu, J. Power Sources, 2012, 198, 423.

$26 \mathrm{H}$. Hu, Z. Zhao, Q. Zhou, Y. Gogotsi and J. Qiu, Carbon, 2012, 50, 3267.

27 Y. M. Shulga, S. A. Baskakov, E. I. Knerelman, G. I. Davidova, E. R. Badamshina and N. Y. Shulga, RSC Adv., 2014, 4, 587.

28 B. Gurzęda, P. Florczak, M. Kempiński, B. Peplińska, P. Krawczyk and S. Jurga, Carbon, 2016, 100, 540.

29 D. Nečas and P. Klapetek, Cent. Eur. J. Phys., 2012, 10, 181.

30 C. Y. Ho and H. W. Wang, Appl. Surf. Sci., 2015, 357, 147.

31 C. M. Chen, Q. Zhang, M. G. Yang, C. H. Huang, Y. G. Yang and M. Z. Wang, Carbon, 2012, 50, 3572.

32 M. A. Pimenta, G. Dresselhaus, M. S. Dresselhaus, L. G. Cançado, A. Jorio and R. Saito, Phys. Chem. Chem. Phys., 2007, 9, 1276.

33 Y. Wang, D. C. Alsmeyer and R. L. McCreery, Chem. Mater., 1990, 2, 557.

34 K. N. Kudin, B. Ozbas, H. C. Schniepp, R. K. Prud'homme, I. A. Aksay and R. Car, Nano Lett., 2008, 8, 36.

35 A. Kaniyoor, T. T. Baby and S. Ramaprabhu, J. Mater. Chem., 2010, 20, 8467.

36 J. Cui, Y. Lai, W. Wang, H. Li, X. Ma and J. Zhan, Carbon, 2014, 66, 738.

37 Y. Hu, S. Song and A. Lopez-Valdivieso, J. Colloid Interface Sci., 2015, 450, 68.

38 Z. González, C. Botas, P. Álvarez, S. Roldán, S. Blanco, R. Santamaría, M. Granda and R. Menéndez, Carbon, 2012, 50, 828.

39 S. Stankovich, D. A. Dikin, R. D. Piner, K. A. Kohlhaas, A. Kleinhammes, Y. Jia, Y. Wu, S. T. Nguyen and R. S. Ruoff, Carbon, 2007, 45, 1558.

40 Z. J. Fan, W. Kai, J. Yan, T. Wei, L. J. Zhi, J. Feng, Y. Ren, L. P. Song and F. Wei, ACS Nano, 2011, 5, 191.

41 E. Paparazzo, Carbon, 2013, 63, 578.

42 Y. Matsumoto, H. Tateishi, M. Koinuma, Y. Kamei, C. Ogata, K. Gezuhara, K. Hatakeyama, S. Hayami, T. Taniguchi and A. Funatsu, J. Electroanal. Chem., 2013, 704, 233.

43 S. Biniak, G. Szymański, J. Siedlewski and A. Świątkowski, Carbon, 1997, 35, 1799.

44 P. Nemes-Incze, Z. Osváth, K. Kamarás and L. P. Biró, Carbon, 2008, 46, 1435. 